\title{
Mechanical Behavior of Articular Cartilage
}

\author{
Nancy S. Landínez-Parra, Diego A. Garzón-Alvarado \\ and Juan Carlos Vanegas-Acosta
}

Additional information is available at the end of the chapter

http://dx.doi.org/10.5772/46881

\section{Introduction}

Articular Cartilage (AC) is a poro-elastic biological material that allows the distribution of mechanical loads and joint movements. As a biphasic material, in the presence of load, the articular cartilage deforms its solid matrix and modifies the fluid hydrostatic pressure within. The aim of this chapter is to present a mathematical model that predicts the mechanical behavior of articular cartilage, taking into account the duality between the solid matrix and articular liquid, and its poro-elastic characteristics. Using a finite element method approach, the response of a piece of articular cartilage in one and two dimensions has been simulated, with tensile, compressive and oscillatory mechanical loads. The analysis of the results allows a qualitative validation of the poro-elastic behavior of the model due to the solid matrix deformation and the fluid outflow that causes variations of pressures inside the articular cartilage in accordance with reported trials. The mathematical model allows for prediction of articular cartilage's biomechanical behavior. These results contribute to the research processes in fields of study such as biomechanics and tissue engineering.

\section{Background}

One of the pathologic entities that most often affect quality of life of individuals is osteoarthrosis (OA), which is caused by the deterioration of cartilage in synovial joints. In the U.S. in the early nineties it was estimated that 37.9 million people (which constituted $15 \%$ of the population) suffered from one of the various existing musculoskeletal diseases. OA was present in 21 million individuals (Lawrence et al., 1998).

OA compromises skeletal muscle function, causing pain and difficulty in basic activities of daily living. Several studies have shown that the forces exerted on cartilage can modify its structure and composition, resulting in a change in the biomechanical behavior of the same (Wu \& Kirk, 2001). The onset and progression of OA are commonly affected by mechanical factors associated with either joint loading or local contact stress (Andriacchi et al., 2004). 
The mechanical environment of cartilage cells (chondrocytes) is an important factor influencing joint health and function. Chondrocytes in articular cartilage utilize mechanical signals in conjunction with other environmental, genetic, extrinsic and intrinsic hormonal and/or paracrine or autocrine factors to regulate their metabolic activity. This capability provides the means by which articular cartilage may alter its structure and composition to meet the physical demands of the body (Boschetti et al., 2004).

The permeability of cartilage contributes to many tissue functions such as transport of food to the chondrocytes, the ability to withstand high loads, and maintaining a fluid film to lubricate opposing joint surfaces (Guilak et al., 1999). Measuring the cartilage permeability considering its depth, by the behavior of a fluid flow induced by the application of a pressure gradient, can show a decrease in permeability in relation to depth for each level of the applied pressure difference.

Here is a review of the anatomy, morphology and physiology of articular cartilage for the purpose of more clearly understanding its responses to load and its relationship to the deformation processes and the changes of fluid pressure within.

\subsection{Biological tissues}

Biological materials are generally multiphase, coexisting in a solid and a fluid phase (Hubertus Frijns, 2000; Doblaré, 2005; Haider \& Schugart, 2006). They also have a strong microstructure that gives them a clear heterogeneous and anisotropic condition and, in addition, its mechanical behavior is strongly nonlinear nonlinear (Wilson et al., 2004; Ateshian et al., 1997; Chan et al., 2004). All of this, without taking into account important aspects such as the strong dependence on age, sex, metabolism, and in particular history and diseases and, ultimately, cell activity and its interaction with the particular environment in which it develops.

In this sense, it can be seen as soft biological tissues in which the elastic modulus is approximately equal to the stresses to which they are subjected. Some typical cases correspond to the arteries and veins, cartilage, ligaments, tendons, muscles or skin. In general, they are composites, made up of organic matrix reinforced by fibers of collagen and elastin. Its behavior again depends on its structural composition, especially the percentage of fibers, their characteristics and the type of grouping. Thus, tissues specialized for tensile strength (e.g. ligaments) are rich in fiber and their orientation essentially coincides with the direction of stress to which they are subjected, while the elastic absorbing the compressive forces (e.g. cartilage) are rich in proteoglycans and fibers distributed in various directions. The cartilage then can be referred to as hydrated tissue, which has a highly compressible behavior corresponding to a saturated biphasic material (if one considers the four-phase ion diffusion) with the possibility of the escape of the fluid inside. The main purpose of this behavior is to provide optimum synovial joint lubrication conditions, friction, wear, shock absorption and load distribution. Interstitial fluid flow in these tissues is crucial both in their viscoelastic properties as well as in the lubrication mechanism; contemplating this is 
necessary to model the behavior of such tissues. Furthermore, they are again heterogeneous and anisotropic due to the preferred orientation of collagen fibers (Wilson et al., 2004).

Understanding the importance of in-depth knowledge of the composition and behavior of articular cartilage, in the next section this tissue will be described.

\subsection{Articular cartilage}

Cartilage is categorized as an avascular, aneural and alinfatic tissue. It's composed of cells called chondrocytes, surrounded by an extracellular matrix (ECM) that they secrete. It's formed by an abundant extracellular matrix in which the chondrocytes are located in spaces called gaps (See Fig 1). The chondrocytes synthesize and secrete the organic components of the extracellular matrix, which are essentially collagen, hyaluronic acid, proteoglycans and glycoproteins. Hyaline and fibrous cartilage are distinguished by the characteristics of the matrix. There is also elastic cartilage, in which the elastin is a part of the extracellular matrix (Koenig, 2011; Sopena-Juncosa et al., 2000; Nordin \& Frankel, 2004).

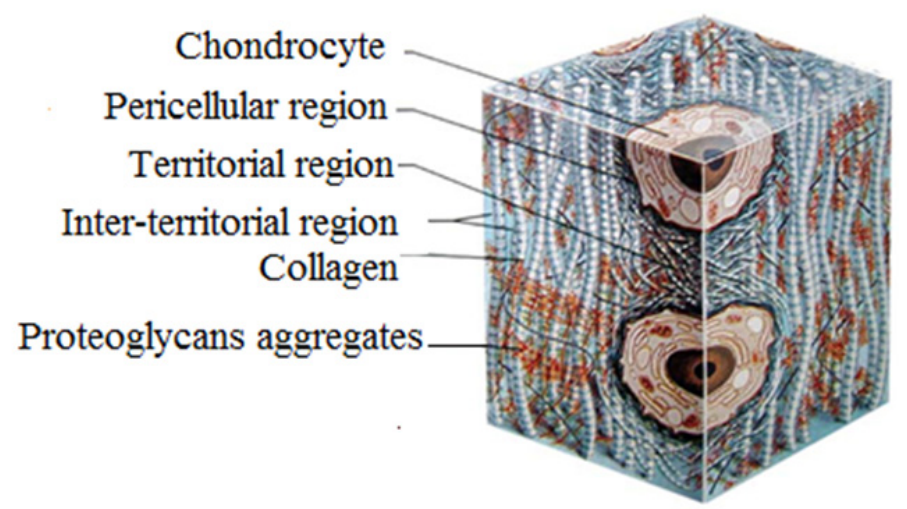

Figure 1. Representation of a fragment of Articular Cartilage. (Sopena-Juncosa et al., 2000)

For a better understanding, each of the structures mentioned above will be defined. Initially chondrocytes will be described, then subsequently the ECM and each of its components.

\subsubsection{Chondrocytes}

They are cells sparsely distributed in the tissue. They constitute approximately 10 percent of cartilage volume. They are located in some gaps within the ECM, to which they adapt. They produce the adjacent ECM, but they are also capable of depolymerizing and removing it to broaden its gaps. (This fact is very evident in the process of endochondral ossification) The key feature of the intercellular substance is its hyper-hydration state ( 80 percent), and combined with proteoglycans, it forms a gel. Greater or lesser elasticity of the cartilage depends on its water content (Koenig, 2011; Sopena-Juncosa et al., 2000; Nordin \& Frankel, 2004). 


\subsubsection{Extracellular Matrix - ECM}

This consists of an organized and dense network of thin collagen fibers embedded in a concentrated solution of proteoglycans. It is responsible for the mechanical properties of cartilage (Koenig, 2011; Sopena-Juncosa et al., 2000; Nordin \& Frankel, 2004). This matrix is composed of:

Water: (60-80 percent) Water is the main component of cartilage, which contributes to its damping properties, cartilage nutrition and the articular lubrication processes. It allows for the deformation of the cartilage in response to mechanical loads, flowing inside and outside them.

Collagen: (10-20percent). Predominantly type II (90-95 percent), giving the cartilage great tensile strength.

Proteoglycans: (PGs) (10-15 percent). These are complex macromolecules, responsible for the resistance to compression of the cartilage. They are secreted by chondrocytes and composed of subunits called glycosaminoglycans (GAGs). The most common GAG is chondroitin-sulfate (of which there are 2 subtypes, the chondroitin-4-sulfate and the chondroitin-6-sulfate), then the keratan-sulphate (or keratin-sulfate) and the dermatansulphate. Chondroitin-4-sulphate is the most abundant and decreases over the years; chondroitin-6-sulfate remains constant; and keratan-sulphate increases with age. The PGs have an average lifespan of three months and have a great capacity for retain water which gives elasticity to the tissue. They are attached to collagen and are responsible for the "porous" structure of cartilage (See Fig.1)

Extracellular Glycoproteins: (anchorite CII, fibronectin, laminin, integrin). They serve a binding function between the ECM and chondrocytes. The most important, integrin, interacts with cell receptors and regulates the migration, proliferation and differentiation of the chondrocytes (Sopena-Juncosa et al., 2000).

At present it is believed that collagen has a different orientation in articular cartilage in relation to its depth, as described by Benninghoff in 1925 in the Arcade model (Wilson, 2005): Packets of primary fibrils extend perpendicular to the sub-chondral bone; the fibrils are separated near the joint surface presented by the curve of the horizontal; each packet of the vertical surface is assumed to be subdivided into two different directions in the curvature of the radial direction (See Fig. 2). It was assumed that the orientation of the secondary fibrils is random and that on the uppermost, fibers are distributed horizontally.

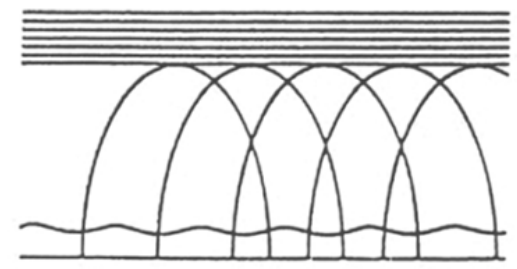

Figure 2. Representation of collagen distribution in articular cartilage. 


\subsection{Architecture of articular cartilage}

Some studies like those reported by Martin, 2002; Sopena et al., 2000; Wilson, 2005 and Meyer \& Wiesmann, 2010; mention that with electron microscopy it can be determined that cartilage has a multilayered arrangement with layers of different thickness and composed of fibrils irregularly cross-linked into a plane parallel to the surface. Using the electron microscope, the following layers of articular cartilage were described:

\subsubsection{Superficial, tangential or sliding layer}

This is adjacent to the joint cavity. The chondrocytes adopt an elongated or ellipsoid shape and they are oriented parallel to the surface. The cells have low activity and poor protein synthesis. This layer possesses few PGs and a high concentration of fine collagen fibers distributed perpendicularly to one another and parallel to the surface in order to withstand the shear forces during joint movement.

\subsubsection{Intermediate or transitional layer}

Cells adopt a rounded morphology and are larger than those of the previous layer. The chondrocytes are irregularly arranged and show a greater presence of PGs and less collagen with thicker fibers arranged obliquely and randomly in all three planes of space. This layer has high metabolic activity and supports compression forces.

\subsubsection{Radial or deep layer}

The cells are rounded and have the same characteristics as layer 2 but adopt a columnar arrangement. They present a high protein synthesis. The collagen fibers are thick and they are distributed parallel to each other and perpendicular to the articular surface to provide resistance to compressive forces. The water content is less than in the previous layers and proteoglycans are most abundant.

\subsubsection{Calcified layer}

This is adjacent to the bone and separated from the previous layer by a basophilic line called tidal or "tidemark", which is a bar wavy tangential arrangement of its fibers and can withstand shear forces. The cells are small and scarce. The matrix is rich in hydroxyapatite crystals. Cartilage anchoring to the sub-chondral bone occurs in this layer (See Fig 3).

The most common tests used for explaining the behavior of articular cartilage under load, expressed in computer models, that include the behavior of swelling or anisotropic properties of the collagen structure for determining the mechanical quality of articular cartilage are: confined compression, the unconfined compression, indentation and swelling (Wilson, 2005). This can be reviewed extensively in the literature. 


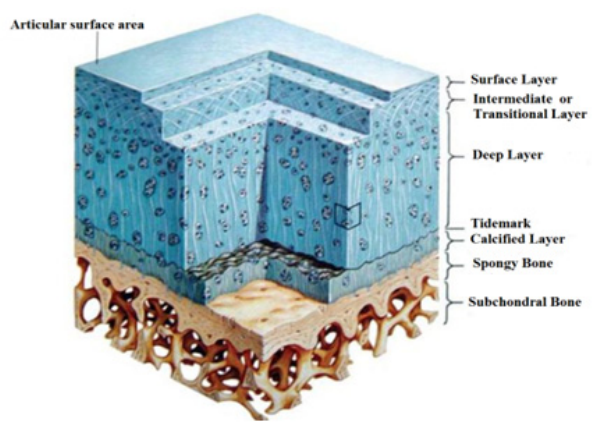

Figure 3. Architectural layout of the articular cartilage according to its various layers. Note the anisotropic distribution of the tissue in relation to the depth thereof. (Sopena-Juncosa et al., 2000)

\subsection{Biphasic behavior of articular cartilage}

Mechanical properties of articular cartilage are attributed to their complex structure and composition of the extracellular matrix that is comprised of a fluid phase (water containing dissolved ions) and a solid matrix that consists mainly of a fibrous network of collagen type II and aggregates of proteoglycans as well as other type of proteins, lipids, and cells (Wilson et al., 2004).

With the mechanical load, the interstitial fluid is redistributed through the pores of the permeable solid matrix, resulting in predominantly viscoelastic behavior (See Fig 4). This highly viscoelastic behavior of articular cartilage is mainly due to two mechanisms: (a) the frictional drag force of interstitial fluid flow through the porous solid matrix (i.e., the flowdependent mechanism), and (b) the function of the time-dependent deformability of the solid matrix (i.e. the flux-independent mechanism) (Garzón, 2007).

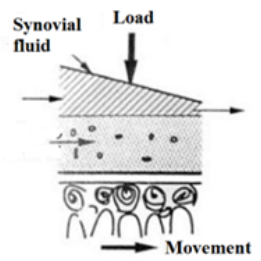

Hydrodynamic

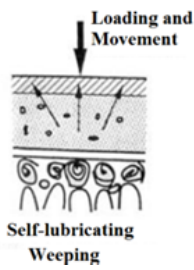

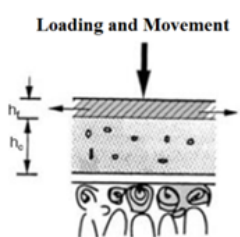

By compressed layer

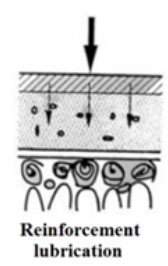

Figure 4. Different forms of lubrication in articular cartilage given by the load applied to the tissue. This lubrication and nutrition takes various forms but mainly by the displacement of the fluid. (Terada et al., 1998). 
Mechanical behavior of cartilage is then described by the biphasic or continuous poro-elastic model that describes the mechanical interactions of the different phases (Donzelli \& Spilker, 1998).

From the mechanical standpoint, the most important components of articular cartilage are strong and highly organized as a network of collagen together with the load of proteoglycans. Due to the fixed charges of proteoglycans, the cation concentration within the tissue is higher than in the surrounding synovial fluid. This excess of ion particles leads to an osmotic pressure difference, which causes swelling tissue. The fibrillar collagen network resists pressure and swelling. This combination makes cartilage a unique, highly hydrated and pressurized tissue, reinforced by the tension of the collagen network (Wilson, 2005).

\subsubsection{Mixtures theory}

Articular cartilage can be described by the mixtures theory as a mixture of four elements: a fibrous network (collagen fibres and proteoglycans), a fluid and a positively and negatively charged particle. However, it's important to differentiate between components and phases. Hubertus, 2000; defines a component as a group of particles with the same properties and a phase as a set of miscible components. Thus in theory the four components can be separated into only two phases: a solid and a fluid phase. In this case the fluid is comprised of three components: the liquid, the cations and anions.

Many authors such as Haider \& Schugart, 2000; Wilson et al., 2004; Haider \& Guilak, 2007; Meng et al., 2002; Wu et al., 1997; Terada et al., 1998; Donzellie et al., 1999 and Donzelli \& Spilker, 1998; among others, have conducted their research, viewing cartilage from this biphasic behavior. This has allowed its analysis as a material with a porous-viscoelastic behavior, in an attempt to better understand its response to loads, forces and overload.

\subsection{Computational analysis of articular cartilage}

Continued use of simulation in medicine has allowed important data to be obtained about the biological, mechanical and chemical behavior of the organs and tissues using mathematical formalization and subsequent numerical simulation of complex biological processes. Various medical problems related to surgery, trauma and rehabilitation have been identified, conceptualized and solved systematically and numerically (Garzón, 2007).

Computer mechano-biology determines the quantitative rules governing actions for cellular expression, differentiation and maintenance of biological and mechanical stimuli, which can be simulated by numerical methods. The procedure for finding such rules is usually through the process of "trial and error" (Van der Meulen \& Huiskes, 2002). The computational tests are simulated usually from problems in the contour value by which the mechanical loads on the boundary are transferred to local mechanical variables (stress and strain). On the biological side, these local mechanical or biophysical variables stimulate cell expression to regulate, for example, the composition of the matrix and the expression of molecular 
substances. Both biological and mechanical parts are combined in a computational model, which considers the application of forces, mechano-transduction, cellular expression, genetics and the transformation of the characteristics of the extracellular matrix. The typical method of numerical implementation of these mechano-biological problems is the finite element method (FEM) (Garzón, 2007).

Finite element computational analysis has been used as an approach to diverse biological processes including the biomechanical behavior of articular cartilage (Wilson et al., 2004; Ateshian et al., 1997; Chan et al., 2004; Wilson, 2005; Donzelli et al, 1999; Donzelli \& Spilker, 1998; Almeida \& Spilker, 1998; Wu \& Herzog, 2000; Levenston et al., 1998). Using the material representation of the continuum phase of cartilage, results have indicated that local intermittent hydrostatic pressure promotes cartilage maintenance (Carter \& Wong, 2003).

\section{Mechanical behavior of articular cartilage (AC):}

Mechanical properties of articular cartilage (AC) are attributed to its complex structure and to composition of its ECM including a fluid phase (water with dissolved ions), and a solid matrix (collagen type II, aggregates of PGs, proteins, lipids, and cells) (Haider \& Guilak, 2007). With the mechanical load, the interstitial fluid is redistributed through the pores of the permeable solid matrix, resulting in predominantly poro-elastic conduct. This behavior of the AC is mainly due to two mechanisms: (a) the frictional force due to drag flow of interstitial fluid through the porous solid matrix (flow-dependent mechanism), and (b) the deformability of the matrix strong function of time (flow-independent mechanism) (Wilson, 2005).

\section{Mathematical model for articular cartilage}

Several authors (Mow, 1980 ; Haider \& Schugart, 2006; Wilson et al., 2005; Haider \& Guilak, 2007; Meng et al., 2002; Wu et al., 1997; Terada et al., 1998; Donzelli et al., 1999; Donzelli \& Spilker, 1998); have conducted their research on cartilage from biphasic behavior that this tissue exhibits. This allowed analysis of the same material as a poro-elastic behavior capable of supporting loads. The mathematical model of the AC as a biphasic material, analyzes the displacement $u(t, x)$ of the solid (matrix) and pressure $p(x)$ of the fluid displaced by the load, thanks to its characteristic of poro-elasticity. This model is described by the equations (1) and (2):

$$
\begin{gathered}
-\nabla \cdot\left(2 \mu_{\mathrm{S}} \underset{=-}{\varepsilon(\mathrm{u})}+\lambda_{\mathrm{S}} \nabla \cdot \underset{-=}{\nabla \mathrm{u})}+\nabla \mathrm{p}=0 \text { en } \Omega\right. \\
\frac{\partial}{\partial \mathrm{t}}(\underset{-}{\mathrm{u}})-\nabla \cdot(\mathrm{k} \nabla \mathrm{p})=0 \text { en } \Omega
\end{gathered}
$$

Equation (1) is derived from the law of conservation of momentum and corresponds to the linear elasticity equation (term 1a) coupled with a term that represents fluid pressure (term $1 b)$. The term $\varepsilon(\mathrm{u})$ corresponds to the strain tensor acting on the $\Gamma$ surface enclosed by $\Omega$. $\mu_{\mathrm{s}} \mathrm{y} \lambda_{\mathrm{s}}$ are the "Lame elastic constants" for the solid, related to Young's modulus and 
Poisson's ratio (E, v). For its part, the equation (2) refers to the change of the dilation of the solid matrix (term 2a) due to the mechanical load created by the divergence of the gradient of the pressure of fluid contained in the domain $\Omega$ (term $2 b$ ) (Frijns, 2000). In this equation, $\mathrm{k}$ is a constant representing the permeability of solid module.

\subsection{Boundary conditions}

Boundary conditions of the model are defined in $\Gamma$ domain and may be dependent on time. The mathematical expression of these conditions is (3-6):

$$
\begin{aligned}
& \mathrm{u}=\mathrm{g}_{\mathrm{u}}^{\mathrm{D}} \text { en } \Gamma_{\mathrm{u}}^{\mathrm{D}} \\
& \mathrm{n} \cdot\left(2 \mu_{\mathrm{s}} \underset{\underline{\underline{ }}}{\varepsilon(\mathrm{u})}+\lambda_{\mathrm{s}} \nabla \cdot \mathrm{uI}_{-=} \mathrm{I}-\mathrm{pI}\right)=\mathrm{g}_{\mathrm{u}}^{\mathrm{N}} \text { en } \Gamma_{\mathrm{u}}^{\mathrm{N}} \\
& \mathrm{p}=\mathrm{g}_{\mathrm{p}}^{\mathrm{D}} \text { en } \Gamma_{\mathrm{p}}^{\mathrm{D}} \\
& -\mathrm{n} .(\mathrm{k} \nabla \mathrm{p})=\mathrm{g}_{\mathrm{p}}^{\mathrm{N}} \text { en } \Gamma_{\mathrm{p}}^{\mathrm{N}} \\
& \text { with } \Gamma=\Gamma_{\mathrm{u}}^{\mathrm{P}}+\Gamma_{\mathrm{u}}^{\mathrm{N}}+\Gamma_{\mathrm{p}}^{\mathrm{D}}+\Gamma_{\mathrm{p}}^{\mathrm{N}}
\end{aligned}
$$

A widely used method for solving partial differential equations in complex geometries is the finite element method (Garzón, 2007). This method allows implementing the numerical model presented in equations (1) and (2) simply and with low computation cost. The method consists of using a vectorial function $W$ or weighting function and a scalar function of $q$, which minimizes the terms of the equations (1) and (2). Multiplying (1) by $W$ and (2) by $q$, and performing integration by parts in the $\Omega$ domain, we obtain a variational of the form (Frijns, 2000):

$$
\begin{aligned}
& a(u, W)+b(W, p)=\left(g_{u}^{N}, W\right) \\
& \frac{\partial}{\partial t} b(u, q)-c(p, q)=\left(g_{p}^{N}, q\right)
\end{aligned}
$$

where:

$$
\begin{gathered}
\mathrm{a}(\mathrm{u}, \mathrm{W})=\int_{\Omega}\left(2 \mu_{\mathrm{s}} \underset{=}{\varepsilon(\mathrm{u})}: \varepsilon(\mathrm{W})+\lambda_{\mathrm{s}}(\nabla \cdot \mathrm{u})(\nabla \cdot \mathrm{W})\right. \\
\mathrm{b}(\mathrm{W}, \mathrm{q})=-\int_{\Omega} \nabla \cdot \mathrm{Wq} \\
\mathrm{c}(\mathrm{p}, \mathrm{q})=\int_{\Omega}(\mathrm{k} \nabla \mathrm{p}) \cdot \nabla \mathrm{q} \\
\left(\mathrm{g}_{\mathrm{u}}^{\mathrm{N}}, \mathrm{W}\right)=\int_{\Gamma_{\mathrm{u}}^{\mathrm{N}}} \mathrm{g}_{\mathrm{u}}^{\mathrm{N}} \cdot \mathrm{W}
\end{gathered}
$$


The solution method is based on dividing the domain $\Omega$ (continuum) in which are defined equations (1) and (2) in their integral form in a series of non-intersecting subdomains $\Omega^{\mathrm{e}}$ called finite elements. The set of finite elements forms a partition of the domain called discretization which together are described by equations (14) and (15):

$$
\begin{gathered}
\mathrm{a}\left(\mathrm{u}_{\mathrm{u}}^{\mathrm{h}}, \mathrm{W}_{\mathrm{h}}^{\mathrm{h}}\right)+\mathrm{b}\left(\mathrm{W}_{\mathrm{h}}^{\mathrm{h}}, \mathrm{p}_{\mathrm{n}}^{\mathrm{h}}\right)=\left(\mathrm{g}_{\mathrm{u}}^{\mathrm{N}}, \mathrm{W}_{\mathrm{h}}^{\mathrm{h}}\right) \\
\mathrm{b}\left(\mathrm{u}_{\mathrm{n}}^{\mathrm{h}}, \mathrm{q}^{\mathrm{h}}\right)-\Delta \mathrm{t} \mathrm{c}\left(\mathrm{p}^{\mathrm{h}}, \mathrm{q}_{\mathrm{n}}^{\mathrm{h}}\right)=\mathrm{b}\left(\mathrm{u}_{\mathrm{n}-1}, \mathrm{q}^{\mathrm{h}}\right)+\Delta \mathrm{t}\left(\mathrm{g}_{\mathrm{p}}^{\mathrm{N}}, \mathrm{q}^{\mathrm{h}}\right)
\end{gathered}
$$

In (15) the $n-1$ subscript indicates the value of a parameter at a $t-1$ time, while the subscript $\mathrm{n}$ indicates the value in the next time, $t_{n}=t_{n-1}+\Delta t$ (Frijns, 2000). Using the Galerkin method, the functions $W$ and $q$ are approximated by the matrix expression (16) where the $N$ vector contains shape functions standard $(\xi, \eta)$ which allow interpolation within each element of the domain.

$$
\begin{aligned}
& \mathrm{q}^{\mathrm{h}}=\mathrm{W}_{\mathrm{h}}^{\mathrm{h}}=\left(\begin{array}{l}
\mathrm{N}_{1} \\
\mathrm{~N}_{2} \\
\mathrm{~N}_{3}
\end{array}\right)=\left(\begin{array}{l}
\frac{1}{2} \xi(\xi-1) \\
(1+\xi)(1-\xi) \\
\frac{1}{2} \xi(\xi+1)
\end{array}\right) \\
& \mathrm{W}_{\mathrm{h}}{ }^{\mathrm{h}}=\left(\begin{array}{l}
\mathrm{N}_{1} \\
\mathrm{~N}_{2} \\
\mathrm{~N}_{3} \\
\frac{1}{4}(1+\xi)(1-\eta) \\
\frac{1}{4}(1+\xi)(1+\eta) \\
\mathrm{N}_{4}
\end{array}\right)=\left(\begin{array}{l}
\frac{1}{4}(1-\xi)(1-\eta) \\
\frac{1}{4}(1-\xi)(1+\eta)
\end{array}\right)
\end{aligned}
$$

In (16), the shape functions correspond to the case of one-dimensional element with three nodes (See Fig. 5a). For two-dimensional case four node elements are used, whose standard shape functions are shown in matrix form (17) and in Figure 5b.

If the integration space $(x, y)$ is changed to $(\xi, \eta)$ by the Jacobian of the transformation and vector notation is adjusted, the equations (1) and (2) can be reduced to a matrix system of elementary type of the form (18) which corresponds to the algebraic discretization in $\Omega \mathrm{e}$ domain of an element, with $k$ elementary stiffness matrix, $m$ the unknowns and $f$ the independent term. Joining the result of (18) for total elements in $\Omega$, a general matrix system is obtained defined as (19) where $K$ is the global matrix of stiffness, $M$ is the vector of unknowns and $F$ is the global vector entries. 


$$
\begin{aligned}
& \mathrm{k} \cdot \mathrm{m}=\mathrm{f} \text { en } \Omega^{\mathrm{e}} \\
& \mathrm{K} \cdot \mathrm{M}=\mathrm{F} \text { en } \Omega
\end{aligned}
$$

a

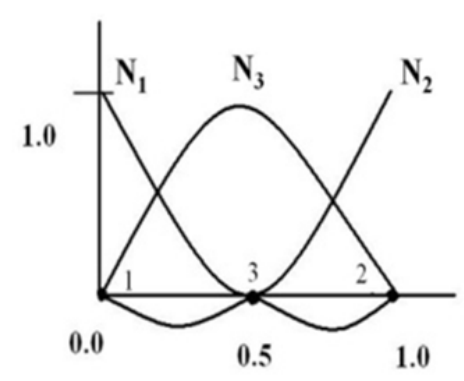

$\mathrm{b}$

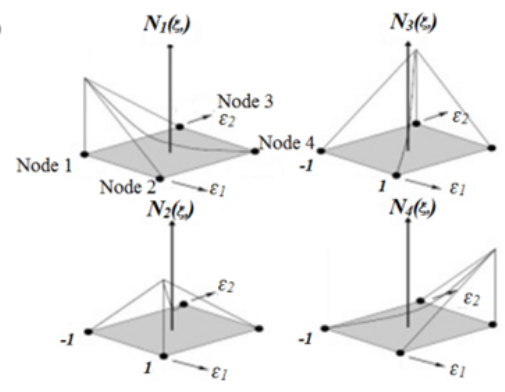

Figure 5. Representation of the shape functions standard. a) Case 1D, 3 nodes per element. b) Case 2D elements with four nodes (Radi, 2007).

\subsection{Model implementation}

Solution of these equations for both $u$ and $p$ are implemented using a routine programmed in Fortran and a desktop PC with 2.4 GHz AMD processor and 1.0 GB of RAM

\subsection{Computer simulation}

Conditions for the simulation were based on experiments by Frijns, 2000 and Wu et al., 1999. Simulations were performed for 1D and 2D looking tissue response to the application of: (a) compression, (b) tensile and (c) oscillating or cyclical loads. The simulation time is 45 seconds of load in all cases. The loads applied in 1D and 2D simulations were performed with the parameters shown in Table 1 . The tests considered the AC as a continuous and homogeneous material with the chondrocytes being part of the continuum.

\begin{tabular}{|c|c|c|c|}
\hline & E & $v$ & $\mathrm{~K}$ \\
\hline Matrix & $0.40 \mathrm{Mpa}$ & 0.1 & $1.0 \times 10^{-2} \mathrm{mmN}^{-1} \mathrm{~s}^{-1}$ \\
\hline Chondrocyte & $1.0 \mathrm{kPa}$ & 0.2 & $1.0 \times 10^{-5} \mathrm{mmN}^{-1} \mathrm{~s}^{-1}$ \\
\hline
\end{tabular}

Table 1. Parameters for the homogeneous material characteristics (Wu et al., 1999).

\subsubsection{Meshing}

For the 1D simulation a mesh was performed that represents a fragment of $0.3 \mathrm{~mm} \mathrm{AC}$. From this mesh 641 nodes and 320 elements of 3 nodes were obtained. In the 2D simulation a mesh was performed that represents a fragment of articular cartilage $0.09 \mathrm{~mm} 2(0.3 \mathrm{~mm}$ x $0.3 \mathrm{~mm}$ ). In this case 10,201 nodes were obtained, equivalent to 10,000 elements of 4 nodes. 


\subsubsection{Boundary conditions}

Simulation was performed so as not to allow displacement at the bottom. Load was applied on the upper edge, allowing the fluid outlet only at the bottom of the tissue fragment, as shown in Figure 6a. For 2D, a condition of tissue confinement was simulated, as shown the Figure $6 \mathrm{~b}$, so as to present only flow at the bottom. The burden was placed at the top and lateral and bottom movement were restricted, similar conditions to those reported in several experimental studies, including that of Ateshian et al, 1997; Frijns, 2000 and Wu et al, 1999.
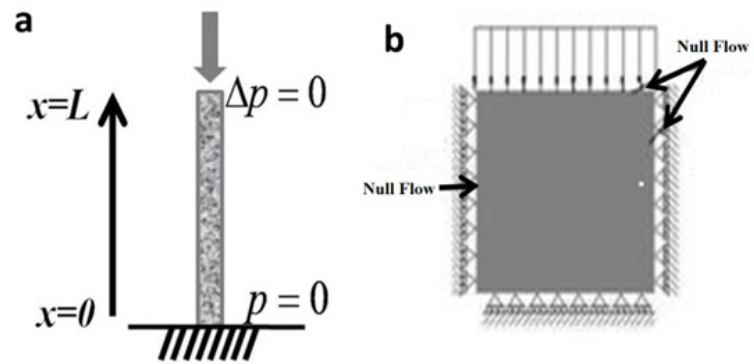

Figure 6. AC scheme for the confinement conditions in (a) 1D and (b) 2D.

\subsubsection{Loading conditions}

Calculations to simulate the applied load were performed from the data for the AC reported by $\mathrm{Wu}$ et al. For the $1 \mathrm{D}$ simulations, a load was applied of $-2.033 \mathrm{~N} / \mathrm{m}$ in compression, 2.033 $\mathrm{N} / \mathrm{m}$ in tension and cyclic loading with frequency equal to $0.1 \mathrm{~Hz}$. In 2D simulations a load was applied on the upper face of cartilage fragment corresponding to the value $-4.0397 \mathrm{e}^{-6}$ $\mathrm{N} / \mathrm{m}$ in compression, $4.0397 \mathrm{e}^{-6} \mathrm{~N} / \mathrm{m}$ in tension. For cyclic loading, it was applied at a frequency equivalent to $0.1 \mathrm{~Hz}$

\section{Results obtained}

\subsection{D simulations}

\subsubsection{Compression loads response}

Responses from the evidence to compressive loads can be seen in Figure 7. Figure 7a shows the negative shift of the solid component that increases its value negatively with increasing loading time. The time initially is zero (0) for $t=0.67 \mathrm{~s}$ and reaching figures of $-15 \times 10^{-4} \mathrm{mmN}-1 \mathrm{~s}^{-1}$ at the time of maximum loading, $t=45 \mathrm{~s}$ for $\mathrm{x}=0.3$. As the liquid flows, the behavior is similar to a linear elastic behavior, because the only component that supports the load is solid.

Figure $7 \mathrm{~b}$ shows the decrease in pressure due to the fluid outlet presented by the compression of the tissue. For an initial time $t=0.67 \mathrm{~s}$ the pressure exerted on the fluid in the tissue is $2 \mathrm{MPa}$, but as the permanence of the compressive, the pressure decreases and takes values close to $0 \mathrm{MPa}$, thereby external pressure is balanced, also zero. 

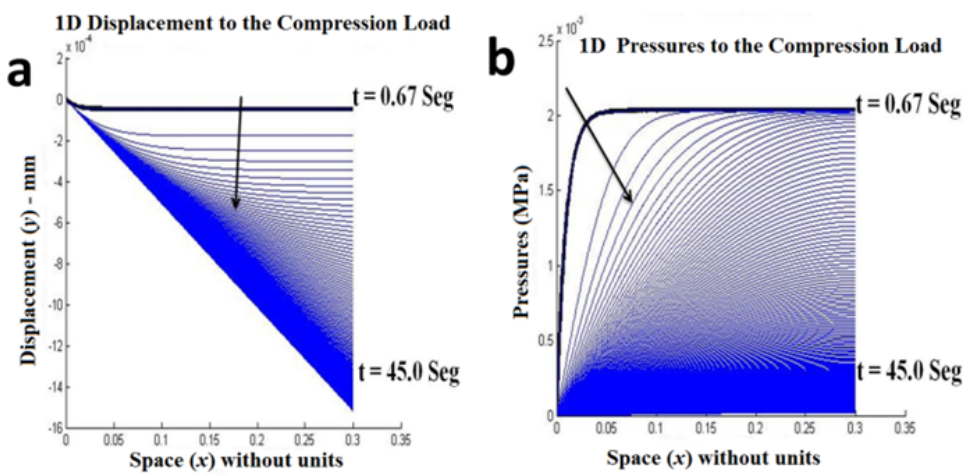

Figure 7. Response of tissue to compression forces. a. Displacement of solid matrix. b. Changes in the fluid pressure in the tissue in presence of the displacement of the same.

\subsubsection{Tensil loads response}

Figure 8 summarizes the results obtained for tensile strength. Figure 8a shows the positive displacement of the solid component in response to tensile load imposed on tissue. The figure shows how the displacements are positive in presence of tension maintained over time. From an initial value of $0 \mathrm{mmN}^{-1} \mathrm{~s}^{-1}$ at $\mathrm{t}=0.67 \mathrm{~s}$, displacement increases up to a maximum of $16 \times 10^{-4} \mathrm{mmN}^{-1} \mathrm{~s}^{-1}$ at a maximum load time $\mathrm{t}=45 \mathrm{~s}$.
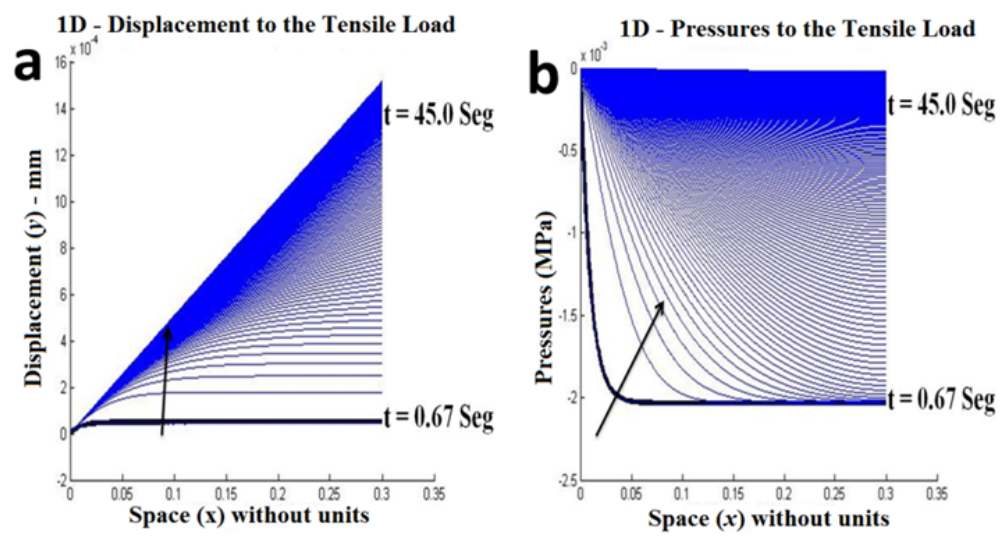

Figure 8. Response of tissue to tensile forces. a. Displacement of solid component tissue upward. b. Change in the fluid pressure generated by the redistribution of fluid in the tissue.

Figure $8 \mathrm{~b}$ shows the increase of fluid pressure generated by the entry thereof into the tissue due to the pressure difference between the internal and external environment (fluid suction). This pressure difference is caused by displacement of the solid component in the positive direction in response to imposed tensile load. It is shown that this progression of the increase of pressure manifests over time, creating a redistribution of the fluid in the tissue. 


\subsubsection{Oscillating loads response}

Responses obtained in the simulation of oscillating charges can be seen in Figure 9. Figure 9a shows the alternating displacement presented at each oscillation.
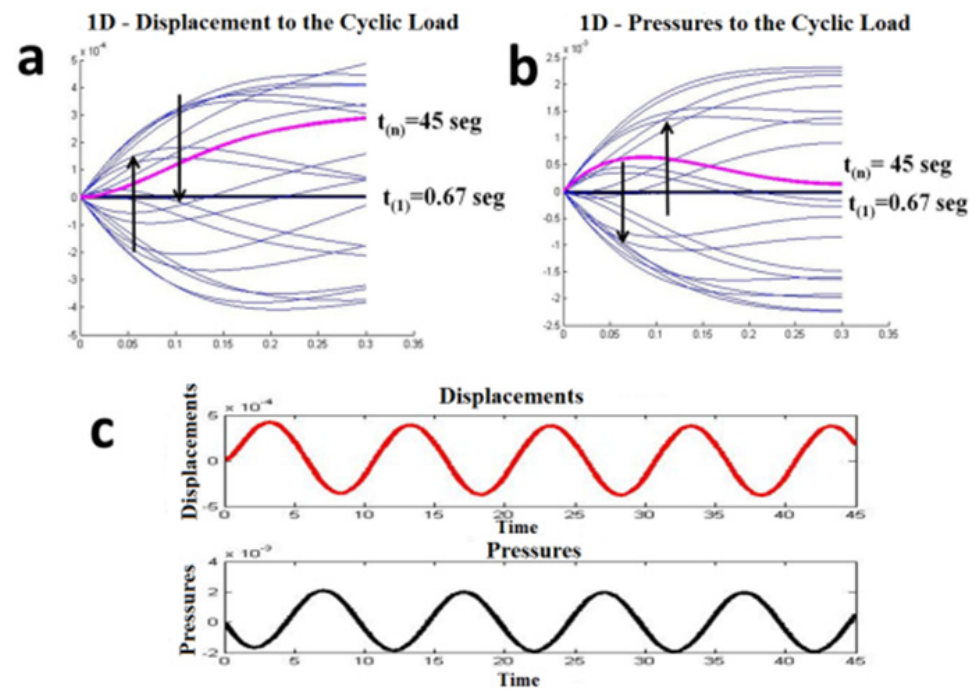

Figure 9. Response to cyclic load. a. Alternate displacement of the solid component. b. Changes in the fluid pressure in oscillate form. c. Delay between the solid deformation and fluid pressure variation.

Initially the movements of the solid are small, close to $0 \mathrm{mmN}^{-1} \mathrm{~s}^{-1}$ for $\mathrm{t}=0.67 \mathrm{~s}$. However, they increase over time reaching a value of $6 \times 10^{-4} \mathrm{mmN}^{-1} \mathrm{~s}^{-1}$ or $-6 \times 10^{-4} \mathrm{mmN}^{-1} \mathrm{~s}^{-1}$, at $\mathrm{t}=45 \mathrm{~s}$, according to the tissue load, tension or compression respectively. Figure $9 \mathrm{~b}$ shows the development of the process of oscillation in the fluid pressures in response to movement that the solid matrix undergoes upon perceiving the cyclic loading. Initially the pressure lowers and then makes an adjustment that increases, being in inverse phase with the evolution of the deformation of the solid phase of the tissue. I.e., once the solid is deformed in the positive direction, the pressure changes, becoming more negative and vice versa.

The obvious alternating deformation processes of the solid in the face of the application of cyclic loads shows that the displacements are caused by the loads exerted on the tissue and the mobilization of fluid from or into the interior as applied tension or compressive loads respectively. These loads, in turn, generate alternation with respect to each instant of time between the variation of the pressure pattern and the variation of the displacement pattern. However, this action is not in complete phase with displacement. One can appreciate the presence and delay of alternating processes described above, because the equation that represents the displacements is elliptical and corresponds to a displacement equation in space while the equation that represents the pressure corresponds to a parabolic equation and represents a much slower diffusion process than the process of displacement (See Fig. 9c). 


\section{2. $2 \mathrm{D}$ simulations}

\subsubsection{Compression loads response}

Figure 10a corresponds to the displacement of the solid in the $y$ axis for each time instant. The displacements are small in the first moments of the load and it can be seen that as the load increases, the time of displacement increases toward more negative values, demonstrating a greater deformation of the solid phase of the tissue. These displacements are produced by the fluid outlet in response to the maintained compression load and it is observed that the greatest displacement occurs in the upper layers of the tissue responsible for receiving the load directly, while the displacement transference is less at a greater tissue depth.

a

\section{COMPRESSION}

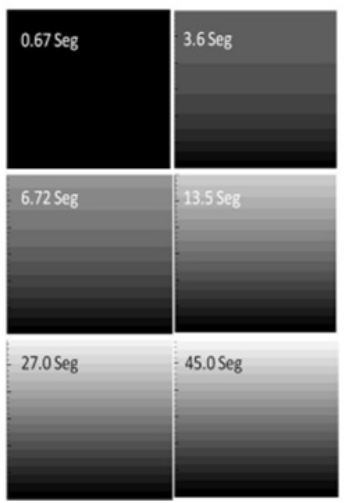

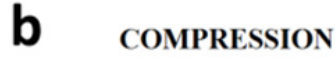
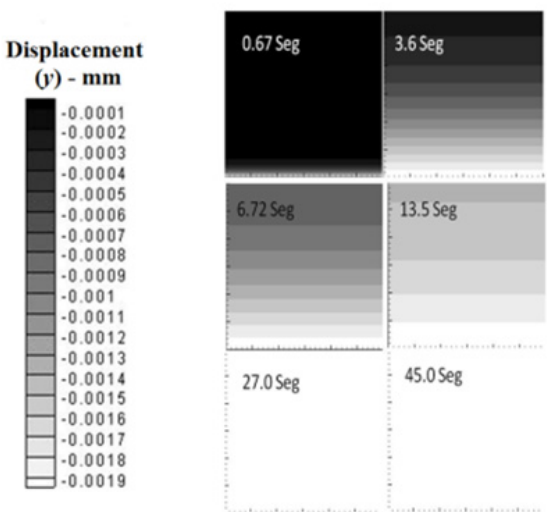

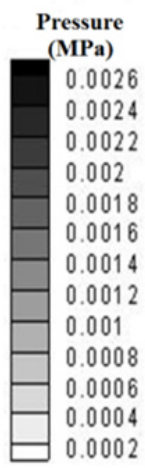

Figure 10. 2D - Response to compressive load. a. $y$ displacements during 45 seconds. b. Behavior of the fluid pressure $p$ due to its outflow in presence of the load.

Figure $10 \mathrm{~b}$ shows the behavior of the fluid pressure at each instant of time. It is observed that the pressure decreases rapidly in all tissue layers reaching values close to $0 \mathrm{MPa}$ in a very short time. This variation of fluid pressure corresponds to the decrease of the same in function of the load exerted over the time and poro-elastic tissue behavior which allows the fluid outlet.

\subsubsection{Tensil loads response}

Results obtained from the tensile simulation are shown in Figure 11. Figure 11a shows the displacement of the solid in the $y$ axis for different time instants. There are major shifts in the early stages of loading $(t=0.67 \mathrm{~s})$ and displacement decreases with increasing load time. It is further noted that with a sustained tensile load at a given time, the greater displacement or elongation occurs in the upper layers of the tissue where the stress is felt in the first instant, which is why the transfer of deformation is smaller at a greater depth of tissue. In this case the fluid pressures tend to increase because the tissue seeks to balance the inside and the outside 
environment. Then displacement occurs in response to stress and produces a reorganization of the fluid within the tissue, interfering with the variation in the pressure thereof.

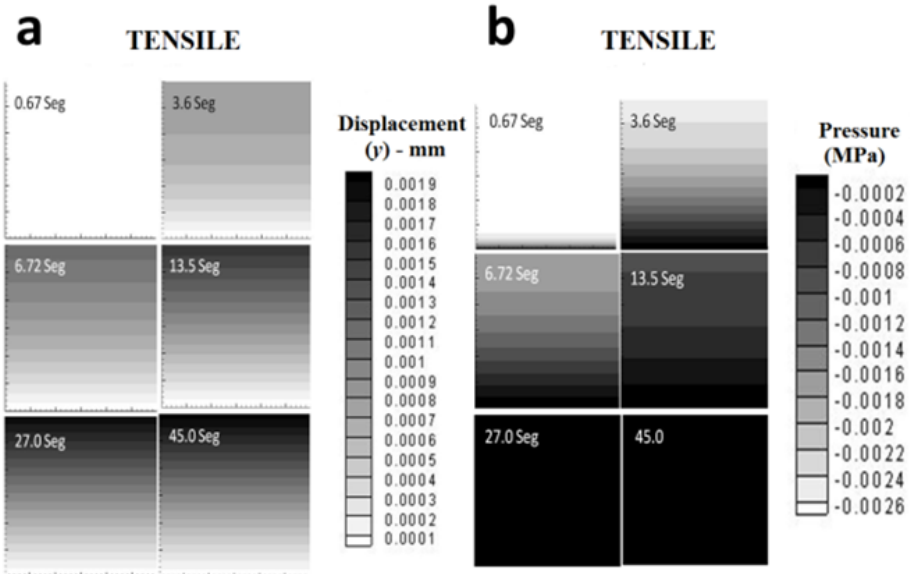

Figure 11. 2D-tensile load response. a. $y$ displacements. b. . Behavior of the fluid pressure $p$ due to its inflow in presence of the load.

Figure $11 \mathrm{~b}$ shows the behavior of the fluid pressure $p$ for different time instants. It is observed that the fluid pressure is increased by small amounts in response to sustained tensile load. This action is due to the compensation of the tissue in response to deformation in elongation by the solid phase of the tissue that requires a redistribution of fluid into the tissue. However, it is evident that at the end of the tensile load time (approximately at $t=$ $27 \mathrm{~s})$, the tissue can't undergo greater deformation in elongation and hence the fluid pressure also tends to stabilize at the inside thereof, causing a steady pressure maintained close to zero which balances with the external pressure.

\subsubsection{Oscillating loads response}

Figure 12a corresponds to the displacement of the solid in the $y$ axis at different time instants. It is noted that the displacements or deformations occur alternately; at the times 1 , 3 and $5(t=0.67 \mathrm{~s}, t=27 \mathrm{~s}=6.57 \mathrm{~s}$ respectively) the displacements are made positive, behavior similar to that observed during exposure to stress loads. Conversely, at the times 2, 4 and 6 $(t=3.37 \mathrm{~s}, \mathrm{t}=16.5 \mathrm{~s}$ and $\mathrm{t}=45 \mathrm{~s}$ respectively) the displacements are negative, consistent with the behavior exhibited by the matrix to compressive loads.

Figure $12 \mathrm{~b}$ shows the behavior of the fluid pressure $p$. Similar to what happened with the deformations of the solid phase, the pressure oscillation in response to cyclic loading imposed on the tissue is evident. Note that at times 1,3 and $5(t=0.67 \mathrm{~s}, t=6.57 \mathrm{syt}=27 \mathrm{~s}$ respectively) the pressure tends to decrease due to the redistribution of fluid in response to perceived stress loads. Because the loads are not maintained, the tissue can't compensate with fluid inlet from the outside which is why the pressure is not increased. Thus, at times 2 , 4 and $6(t=3.37 \mathrm{~s}, \mathrm{t}=16.5 \mathrm{~s}$ and $\mathrm{t}=45 \mathrm{~s}$ respectively), the pressure tends to increase in 
response to the compression of the tissue. This is due to the rapidity with which compressive and tensile loads are alternated, loads which prevent the behavior of cartilage during the cyclic loading from exhibiting the behavior that corresponds exclusively to the case of tension or compression.
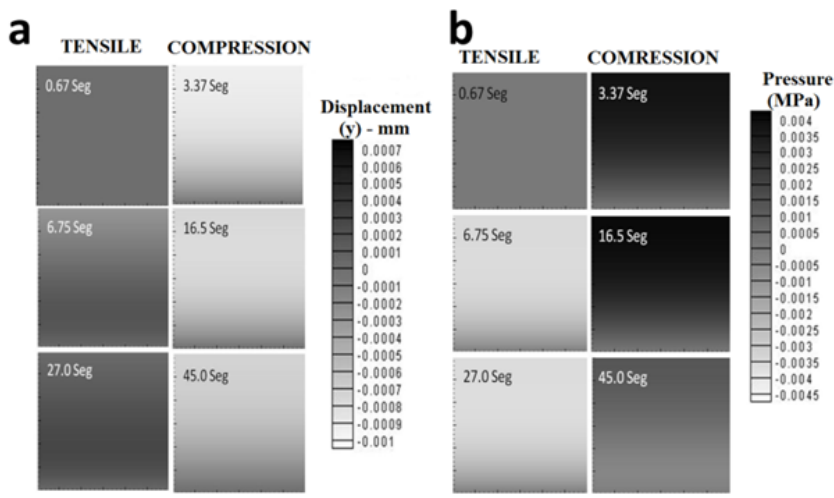

Figure 12. 2D - Response to oscillate load. a. $y$ displacements. b. Oscillation of the pressure $p$ within the tissue according to the load.

\section{Final discussion}

There are several theories explaining the behavior of AC in the presence of load conditions, summarized in computational models that include the swelling process and the properties of the anisotropic structure of collagen. The most frequently used tests to determine the mechanical qualities of the AC are the confined compression, the unconfined compression, the indentation and the swelling tests (Wilson, et al., 2005), carried out using numerical approximation tools.

For purposes of meeting the stated objectives, we simulated a condition of confinement of the tissue that allows the flow at the bottom to restrict lateral and bottom movement. Conditions were similar to those reported in practical experiments as the papers presented by Ateshian et al., 1997; Frijns, 2000 and Wu et al., 1999; among others. The data obtained from the simulations confirm the theory of biphasic articular cartilage, first proposed by Mow et al., 1980; and supported by several authors as Haider et al., 2006; Wilson et al., 2005, Haider \& Guilak , 2007; Meng et al., 2002; Wu et al., 1997, Terada et al., 1998, Donzelli et al., 1999 and Donzelli \& Spilker, 1998; among others.

Results allow us to conclude that articular cartilage exhibits a displacement response of the solid component (matrix) and a variation in the pressure of fluid component due to the exit or entrance thereof, with decreases in pressure in response to compressive loads and increases at the same tensile loads. The displacement is caused by outflow of fluid in response to the maintained compressive load. However it is important to note that once the tissue reaches its maximum displacement, it behaves as a solid rather than as a poro-elastic material. From this point the fluid can't flow out of the tissue because the pressure is balanced with the external fluid begins to bear part of the load. 
If this pressure is maintained for prolonged periods dehydration of the tissue may result and cause changes in normal behavior, making it temporarily or permanently more sensitive to injury. These findings support the conclusion that a load consistently maintained for long periods of time or an excessive load that exceeds the characteristics of the tissue once it has reached the maximum possible displacement, can make one more vulnerable to overuse injuries.

The data reported are the beginning of broader work in the study of cartilaginous tissues which can incorporate the cellular component differentially and cartilage's own biochemistry in the model. The results obtained motivate the efforts that currently seek to simulate the production and destruction of the matrix in the presence of mechanical loads, to simulate the restructuring of the same after an injury, to apply mathematical models in the study of cartilage growth and to study their behavior in vitro and in vivo. These lines of research aim to provide a solid foundation for the development of AC experiments in vivo and in vitro that expands the range of applications of numerical simulation techniques and techniques used in tissue engineering.

\section{Author details}

Nancy S. Landínez-Parra

Group of Mathematical Modeling and Numerical Methods GNUM-UN, Faculty of Engineering, National University of Colombia, Colombia

Human Corporal Movement Department, Faculty of Medicine, National University of Colombia, Colombia

Diego A. Garzón-Alvarado and Juan Carlos Vanegas-Acosta

Group of Mathematical Modeling and Numerical Methods GNUM-UN, Faculty of Engineering, National University of Colombia, Colombia

\section{Acknowledgement}

The authors wish to thank the Research Division of Bogota (DIB) of the National University of Colombia in the Call for Research for supporting this work under the project "Mathematical Modeling and Simulation of Processes in Mechanical and Biomedical Engineering" and Colciencias 2011 throughout the project "Model for the definition of term of the Layout of a manufacturing cell through set theory and optimization" who contributed to the financing the chapter.

\section{References}

Almeida, E.S. \& Spilker, R.L. (1998). Finite element formulations for hyperelasticbiphasic soft tissues transversely isotropic. Computer methods in applied mechanics and engineering. 151, 513-538 
Andriacchi, T.P., Mûndermann, A., Smith, R.L., Alexander, E.J., Dyrby, C.O. \& Koo, S. (2004). A framework for the in vivo pathomechanics of osteoarthritis at the knee. Annals of Biomedical Engineering. 32, 447-457.

Ateshian, G.A., W.H. Warden, J.J. Kim, R.P. Grelsamer \& V.C. Mow. (1997). Finite deformation biphasic material propertieso f bovine articular cartilage from confined compression experiments. J. Bimechanics. Vol 30. Nos. 11/ 12. pp 1157-1164. Published by Elsevier Science

Boschetti, F., Miotti, C., Massi, F., Colombo, M., Quaglini, V., Peretti, G.M. \& Pietrabissa, R. (2002). An Experimental Study on Human Articular Cartilage Permeability. Proceedings of the Second Joint EMBS/BMES Conference. Houston, TX, USA. October 2002. 23-26

Carter, D.R. \& Wong, M. Modelling Cartilage Mechanobiology. (2003). The Royal society. Philos Trans R Soc Lond B Biol Sci. 2003 September 29; 358(1437): 1461-1471.

Chan, B., Donzelli, P.S. \& Spilker, R.L. (2000). A Mixed-Penalty Biphasic Finite Element Formulation Incorporating Viscous Fluids and Material Interfaces. Annals of Biomedical Engineering, Vol. 28, pp. 589-597

Doblaré Castellano, M. (3 de noviembre del año 2005). Sobre el Modelado en Biomecánica y Mecano-biología. Discurso de ingreso a la Real Academia de Ciencias Exactas, Físicas, Químicas y Naturales de Zaragoza.

http://www.unizar.es/acz/02AcademicosNumerarios/Discursos/Doblare.pdf

Donzelli, P.S., Spilker, R.L., Ateshian, G.A. \& Mow, V.C. (1999) Contact analysis of biphasic transversely isotropic cartilage layers and correlations with tissue failure. Journal of Biomechanics. 32, 1037-1047.

Donzelli, P.S. \& Spilker, R.L. (1998). A contact finite element formulation for biological soft hydrated tissues. Computer methods in applied mechanics and engineering. 153, 63-79

Frijns, A.J.H. (2000). A Four-Component Mixture Theory Applied to Cartilaginous Tissues. Tesis Doctoral. Eindhoven University of Technology.

Garzón, D.A. (Mayo de 2007). Simulación de Procesos de Reacción-Difusión: Aplicación a la Morfogénesis del Tejido Óseo. Tesis doctoral. Centro Politécnico Superior de la Universidad de Zaragoza. Zaragoza.

Guilak, F., Jones, W., Ting-beall, P. \& Lee, G. (1999). The deformation behavior and mechanical properties of condrocitos in articular cartilage. Osteoarthritis and Cartilage. 7 , 1: 59-70. January 1999

Haider, M.A. \& Schugart, R.C. (2006). A numerical method for the continuous spectrum biphasic poroviscoelastic model of articular cartilage. Journal of Biomechanics. 39, 177-183.

Haider, M. A. \& Guilak, F. (2007). Application of a three-dimensional poro-elastic BEM to modelling the biphasic mechanics of cell-matrix interactions in articular cartilage. Computer methods in applied mechanics and engineering. 196, 2999-3010.

Koenig, C. (Noviembre de 2011). Curso de Histología. Oficina de Educación Médica. Escuela de Medicina. Pontificia Universidad Católica de Chile. Consultado el 25 Noviembre 2011. http//escuela.med.puc.cl

Lawrence, R.C., Helmick, Ch.G. \& Arnett, F.C. (1998). Estimates of the prevalence of the arthritis and selected musculoskeletal disorders in the United States. Arthritis Rheum; 41: 2213-2218.

Levenston, M.E., Frank, E.H. \& Grodzinsky, A.J. (1998). Variationally derived 3-field finite element formulations for quasistaticporo-elastic analysis of hydrated biological tissues. Computer methods in applied mechanics and engineering. 156, 231-246. 
Martín-Hernández, C. (2002). Estudio Mecánico, Histológico, e Histo-morfométrico del Regenerado de Cartílago a Partir de Injertos de Periostio Invertido. Tesis Doctoral. Universidad Autónoma de Barcelona.

Meng, X.N., Leroux, M.A., Laursen, T.A. \& Setton, L.A. (2002). A nonlinear finite element formulation for axisymmetric torsion of biphasic materials. International Journal of Solids and Structures. 39, 879-895.

Meyer, U. \& Wiesmann, H.P. (April 11, 2006). Bone and Cartilage Engineering. (1 edition) ED Springer. ISBN 978-3-540-25347-1. Germany. pp 25-27.

Mow, V.C., Kuei, S.C., Lai, W.M. \& Armstrong, C.G. (2006) Biphasic creep and stress relaxation of articular cartilage in compression: theory and experiments. Journal of Biomechanical Engineering. 102: 73-84. X

Nordin M. \& Frankel, V. (2004). Biomecánica Básica del sistema Musculoesqueletico. ED McGrawn-Hill Interamericana. pp 61.

Radi, M. (1998). Three-Dimensional Simulation of Thermal Oxidation. Dissertation. Institute for Microelectronics. Faculty of Electrical Engineering and information technology 1998. Consultado el 25 de Abril de 2012. http://www.iue.tuwien.ac.at/phd/radi

Sopena-Juncosa, J.J., Carrillo-Poveda J.M., Rubio-Zaragoza M., Redondo-García J. I., SerraAguado I. \& Soleri-Canet I. (2000). Estructura y función del cartílago articular. Portada: Armas Frente a la Patología Articular.

Terada, K., Ito, T. \& Kikuchi, N. (1998). Characterization of the mechanical behaviors of solid-fluid mixture by the homogenization method. Computer methods in applied mechanics and engineering. 153, 223-257.

Van der Meulen, M. \& Huiskes, R. (2002). Why Mecanobiology? A survey article. Journal of Biomechanics. Volume 35, Issue 4, Pag: 401-414.

Wilson, W., Van Donkelaar, C.C., Van Rietbergen, B., Itoa, K. \& Huiskes, R. (2004). Stresses in the local collagen network of articular cartilage: a poroviscoelastic fibril-reinforced finite element study. Journal of Biomechanics. 37, 357-366.

Wilson, W., Van Donkelaar, C.C., Van Rietbergen \& Huiskes, R. (2005). A fibril-reinforced poroviscoelastic swelling model for articular cartilage. Journal of Biomechanics. 38, 11951204.

Wilson, W. (2005). An explanation for the onset of mechanically induced cartilage damage. Tesis Doctoral. Technische Universite it Eindhoven. Eindhoven.

Wu, J.Z., Herzog, W. \& Epstein, M. (1997). An improved solution for the contact of two biphasic cartilage layers. Journal of Biomechanics. Vol. 30, No. 4, pp 371.-375

Wu, J.Z., Herzog, W. \& Epstein, M. (1999). Modelling of location- and time-dependent deformation of chondrocytes during cartilage loading. Journal of Biomechanics. 32: 563-572.

Wu J.Z. \& Herzog, W. (2000). Finite Element Simulation of Location and Time-Dependent Mechanical Behavior of Chondrocytes in Unconfined Compression Tests. Annals of Biomedical Engineering. Vol. 28, pp. 318-330

Wu J.P. \& Kirk, T.B. (November 2001). A Study of the Shape Change of the Sheep Chondrocytes with Application of Compression to Cartilage. Seventh Australian and New Zealand Intelligent Information Systems Conference, 18-21. Perth, Western Australia 